\title{
SPACE-CHARGE DRIVEN EMITTANCE GROWTH IN A 3D MISMATCHED ANISOTROPIC BEAM
}

\author{
J. Qiang*, R. D. Ryne, LBNL, Berkeley, CA 94720, USA \\ I. Hofmann, GSI Darmstadt, Planckstrasse 1, 64291 Darmstadt, Germany
}

\begin{abstract}
In this paper we present a 3D simulation study of the emittance growth in a mismatched anisotropic beam. The equipartitioning driven by a 4th order space-charge resonance can be significantly modified by the presenc of mismatch oscillation and halo formation. This causes emittance growth in both the longitudinal and transverse directions which could drive the beam even further away from equipartition. The averaged emittance growth per degree of freedom follows the upper bound of the 2D free energy limit plus the contributions from eqipartitioning.
\end{abstract}

\section{INTRODUCTION}

Emittance growth is one of the most fundamental issues in accelerator beam dynamics studies. In a constant focusing channel (smooth approximation of the real machine), the energy anisotropy of the beam is defined as the ratio of the longitudinal temperature to transverse temperature, $\frac{T_{z}}{T_{x}}=\frac{\epsilon_{z} k_{z}}{\epsilon_{x} k_{x}}$, where $z$ is correlated to the longitudinal direction and $x$ is the transverse direction. For a collisionless anisotropic beam in the accelerator, the nonlinear space-charge forces coupling the longitudinal and transverse directions cause emittance exchange among different degrees of freedom when the internal resonance conditions are satisfied. This will be further enhanced by the collective space-charge instability which can grow out of the noise in an anisotropic beam. In this case, the emittance grows in a plane that receives energy, and decreases in a plane that loses energy. A major fourth order difference resonance band in the vicinity of the symmetric focusing has been identified and studied $[1,2,3,4]$. It was found that within this fourth order resonance band, the anisotropic beam will approach to equipartition within a few betatron oscillation periods. Outside the stopband of the coupling resonance, the emittance exchange is small. An initial anisotropic beam can remain anisotropic for a long time without progressing to an equipartitioned beam.

On the other hand, in accelerators, the change of focusing lattice can cause the mismatch between the input beam and the transport system. The mismatch will result in the oscillation of beam envelope and excite the envelope eigenmodes. These envelope modes possess additional free energy compared with the stationary distribution. Individual particles with right oscillation frequency can resonate with these envelope modes through the so-called parametric resonance, e.g. $2: 1$ resonance, and attain large amplitude to form halo $[5,6]$. These halo particles extract the energy

\footnotetext{
*jqiang@lbl.gov
}

from the envelope modes and convert the free energy from mismatch into thermal energy, which causes beam emittance growth.

In recent years, studies have been focused on the understanding of the emittance exchange of an rms matched beam with initial energy anisotropy $[2,3,4,7,8]$ or the mismatched halo formation without the presence of energy anisotropy [9]. For a mismatched anisotropic beam, the final rms emittance growth is more complicated depending on the longitudinal to transverse tune ratio and the longitudinal to transverse emittance ratio of the beam. In this paper, we have carried out a detailed parameter study of the final emittance growth in a constant focusing channel as a function of longitudinal to transverse tune ratio for two longitudinal to transverse emittance ratios. The simulations have been done using the 3D particle-in-cell code IMPACT [10]. In the simulations, which include a selfconsistent space-charge calculation, we used one million particles on a $64 \times 64 \times 64$ Cartesian grid. We have assumed that in the 3D simulation the initial distribution in $y$ is identical to that in $x$. The numerical convergence of the simulation has been checked using a larger number of macroparticles, more grid points, and a smaller step size.

\section{SPACE-CHARGE DRIVEN EMITTANCE GROWTH}

We first discuss the emittance growth without the presence of the major fourth order coupling resonance in a mismatched beam. In this case, we have chosen $\epsilon_{z} / \epsilon_{x}=1$, $k_{x} / k_{0 x}=0.6$ and a symmetric mismatch factor of 1.3 in all three directions. The emittance exchange around the fourth order difference resonance $2 k_{z}-2 k_{x} \approx 0$ is negligible since there is no free energy available to transfer for a beam with equipartitioned temperature ratio $T_{z} / T_{x}=1$. Fig. 1 shows the final relative rms emittance growth as a function of $k_{z} / k_{x}$ for fixed transverse tune depression. The simulations were done through 100 zero current betatron oscillation periods to reach saturated amplitudes. The initial distribution is a 6D Gaussian distribution. For $0.56<$ $k_{z} / k_{x}<1$, the relative emittance growth in the transverse direction is larger than that in the longitudinal direction. Above $k_{z} / k_{x}=1$, the emittance growth in the longitudinal direction becomes dominant. In both cases, the emittance growth is predominately in the direction with stronger focusing. Such anisotropic emittance growth could make the beam move further away from equipartition. It has been shown by Franchetti et. al. that around $k_{z} / k_{x}=1$, with stronger focusing in that plane, the fixed point in that plane 


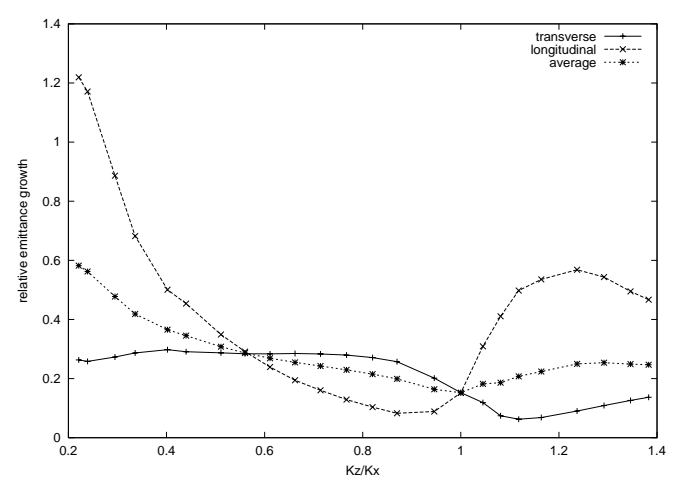

Figure 1: Final relative rms emittance growth in the longitudinal direction, transverse direction, and averaged per degree of freedom as a function of tune ratio $k_{z} / k_{x}$ $\left(k_{x} / k_{x 0}=0.6, \epsilon_{z} / \epsilon_{x}=1\right)$ for an initial mismatched ( $M=1.3)$ Gaussian distribution.

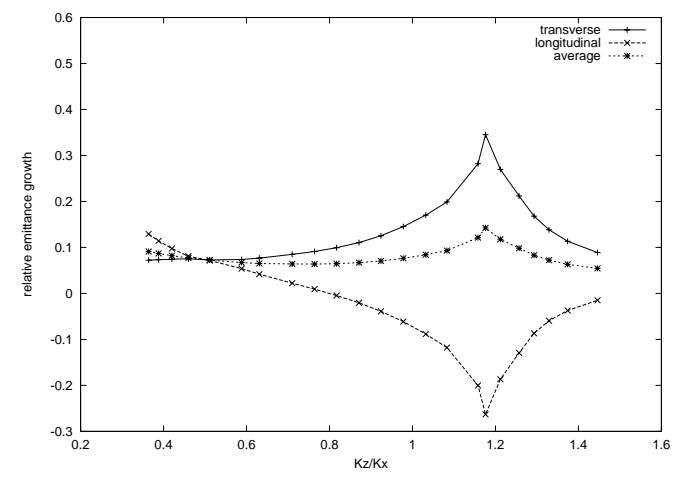

Figure 2: Final relative rms emittance growth in the longitudinal direction, transverse direction, and averaged per degree of freedom as a function of tune ratio $k_{z} / k_{x}$ $\left(k_{x} / k_{x 0}=0.6, \epsilon_{z} / \epsilon_{x}=2\right)$ for an initial matched Gaussian distribution.

for the typical $2: 1$ parametric resonance moves closer to the core [9]. This results in more particles being involved in the parametric resonance in that plane and larger emittance growth. Additional final emittance growth in the longitudinal direction is observed for $0.2<k_{z} / k_{x}<0.56$. The large final longitudinal emittance growth is associated with the contributions from the halo formations and from the equipartitioning driven by the higher order modes.

Next, we are going to discuss the emittance growth of an anisotropic beam with the presence of the major fourth order coupling resonance. Within the resonance band of $2 k_{z}-2 k_{x} \approx 0$, there will be pronounced emittance exchange between the transverse direction and the longitudinal direction even though the beam is initially rms matched. Fig. 2 shows the final relative emittance growth as a function of the longitudinal to transverse tune ratio for an initial rms matched Gaussian beam with $k_{x} / k_{x 0}=0.6$ and $\epsilon_{z} / \epsilon_{x}=2$. Within the resonance band $2 k_{z}-2 k_{x} \approx 0$, the maximum longitudinal emittance decreases by about $26 \%$, while the maximum transverse emittance increases

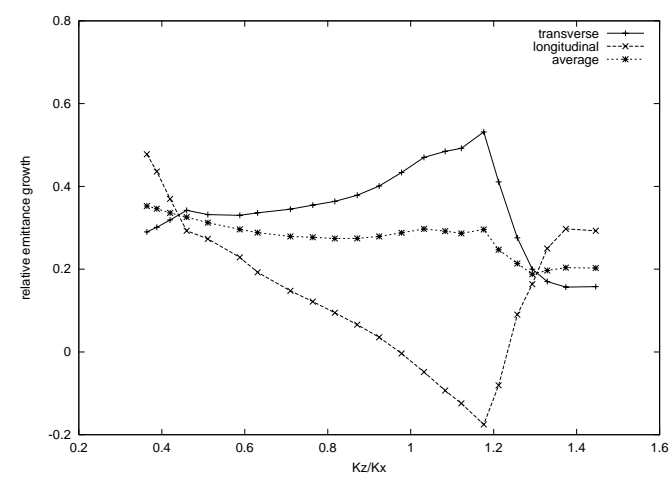

Figure 3: Final relative rms emittance growth in the longitudinal direction, transverse direction, and averaged per degree of freedom as a function of tune ratio $k_{z} / k_{x}$ $\left(k_{x} / k_{x 0}=0.6, \epsilon_{z} / \epsilon_{x}=2\right.$, and $\left.M=1.3\right)$ for an initial Gaussian distribution.

by about $34 \%$. The energy anisotropy $T_{z} / T_{x}$ has dropped from initial 2.3 to 1.1 at the peak of resonance.

With the presence of initial mismatch in above anisotropic beam, the final emittance growth within the fourth order resonance band $2 k_{z}-2 k_{x} \approx 0$ for the rms matched beam is significantly modified. The mismatch causes the envelope oscillations and halo formation in both transverse and longitudinal directions. As a result, the emittances grow in both directions even with the presence of initial emittance exchange. This process pushes the final state of the mismatched anisotropic beam away from equipartition. Fig. 3 shows the final relative emittance growth as a function of $k_{z} / k_{x}$ for an initial Gaussian distribution with a fixed $k_{x} / k_{x 0}=0.6, \epsilon_{z} / \epsilon_{x}=2$, and an initial 1.3 mismatch in all three directions. The mismatched anisotropic beam shows both equipartitioning and anisotropic emittance growth. Comparing with the case of emittance growth around $2 k_{z}-2 k_{x} \approx 0\left(0.6<k_{z} / k_{x}<\right.$ 1.4) for a mismatched beam with $\epsilon_{z} / \epsilon_{x}=1$, there exists a regime of equipartitioning $\left(0.97<k_{z} / k_{x}<1.24\right)$ for the mismatched beam with $\epsilon_{z} / \epsilon_{x}=2$. Comparing with the matched anisotropic beam with $\epsilon_{z} / \epsilon_{x}=2$, the peak of the resonant emittance exchange occurs at the same tune ratio. However, the range of tune ratio for the final emittance exchange has been reduced from $0.8<k_{z} / k_{x}<1.45$ to $0.97<k_{z} / k_{x}<1.24$. Within this range, the fourth order resonance driven equipartitioning is stronger than the halo driven emittance growth. There is a net final emittance exchange between longitudinal direction and transverse direction. Outside this range, the opposite is true. The emittance growth from the mismatched halo overcomes the initial equipartitioning process. The final state of the beam can be driven further away from equipartition.

Even though the emittance growth along the transverse and longitudinal direction shows strong dependence on the longitudinal to transverse tune ratio, the averaged rms emittance growth per degree of freedom, is found to be relatively insensitive to the ratio of the tune within the range 


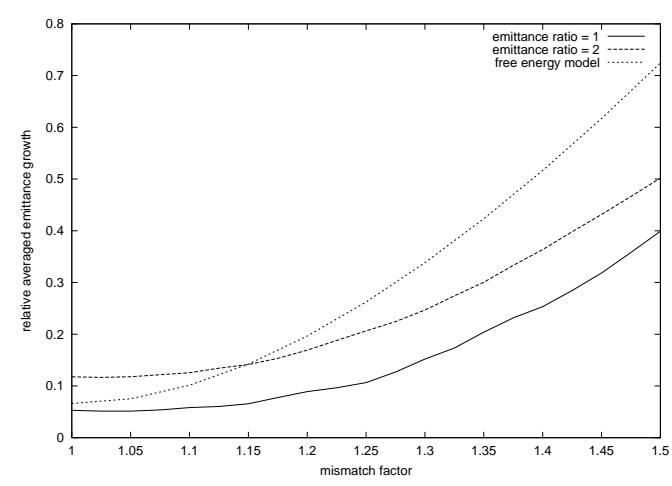

Figure 4: Relative averaged rms emittance growth with $\epsilon_{z} / \epsilon_{x}=1, \epsilon_{z} / \epsilon_{x}=2$, and compared with free energy theory, as a function of mismatch factor for Gaussian beams $\left(k_{x} / k_{x 0}=0.6, k_{z 0} / k_{x 0}=1.0\right)$.

$0.6<k_{z} / k_{x}<1.4$ as shown in Figs. 1-3. This makes it feasible to estimate the average emittance growth and to compare with the "free energy" limit derived by Reiser for symmetrically focused beam [11]. The latter is a " $2 \mathrm{D}$ " approximation understood as the maximum possible rms emittance growth, if all of the energy added to an axially symmetric beam by radial mismatch is "decohered" and a new matched uniform beam is obtained - regardless of the actual driving mechanism. Fig. 4 shows the averaged rms emittance as a function of mismatch factor for an initial Gaussian beam with $k_{z 0} / k_{x 0}=1, \epsilon_{z} / \epsilon_{x}=1$ and 2 together with the emittance growth calculated from the free energy model. The emittance growth using the $2 \mathrm{D}$ free energy model includes the contributions from the initial charge redistribution of the Gaussian beam and from the initial envelope mismatch. For $\epsilon_{z} / \epsilon_{x}=1$, the averaged emittance growth per degree of freedom from simulation rises slower than that from the free energy model as the mismatch factor increases. For $\epsilon_{z} / \epsilon_{x}=2$, the averaged emittance growth from simulation is larger than that from the free energy model for mismatch factor less than 1.15. This extra-emittance growth is due to contributions from equipartitioning. For mismatch factor greater than 1.15 , the emittance growth calculated from the free energy model quickly takes over. The general function dependence of the averaged emittance growth on the mismatch factor for $\epsilon_{z} / \epsilon_{x}=2$ and $\epsilon_{z} / \epsilon_{x}=1$ is similar except an upshift for the $\epsilon_{z} / \epsilon_{x}=2$ case due to the equipartitioning within the coupling resonance. The smaller averaged emittance growth from simulations is due to the fact that there is an incomplete transfer of free energy of envelope oscillation to the emittance growth. The free energy model tends to overestimate the emittance growth since it does not take into account the field energy associated with nonuniform density in the final stationary distribution. Including the contributions from the energy anisotropy, the 2D free energy model represents an upper bound for the averaged emittance growth per degree of freedom in a mismatched anisotropic beam.

\section{CONCLUSIONS}

In summary, the final equipartitioning state of an anisotropic beam within the fourth order coupling resonance has been significantly modified with the presence of the rms mismatch. The anisotropic beam within the resonance band can be pushed further away from the equipartition by the mismatch halo induced emittance growth. For a mismatched anisotropic beam, the emittance growth shows a superposition of the contribution from the equipartitioning and from the mismatched halo formation. Even though the emittance growth along the transverse and longitudinal directions shows strong dependence on the longitudinal to transverse tune ratio, The averaged rms emittance growth per degree of freedom is relatively insensitive to the tune ratio and follows the bound of free energy model plus the equipartitioning contributions.

\section{ACKNOWLEDGMENTS}

The research used resources of the National Energy Research Scientific Computing Center, which is supported by the Office of Science of the U.S. Department of Energy (US DOE/SC) under Contract No. DE-AC03-76SF00098. This work was performed under the auspices of a Scientific Discovery through Advanced Computing project, "Advanced Computing for 21st Century Accelerator Science and Technology, which is supported by the US DOE/SC Office of High Energy and Nuclear Physics and the Office of Advanced Scientific Computing Research.

\section{REFERENCES}

[1] B. W. Montague, CERN Report No. 68-38, 1968.

[2] R. A. Kishek, P. G. O'Shea, and M. Reiser, Phys. Rev. Lett. 85, 4514 (2000).

[3] I. Hofmann, J. Qiang, and R. Ryne, Phys. Rev. Lett. 86, 2313 (2001).

[4] I. Hofmann and O. Boine-Frankenheim, Phys. Rev. Lett. 87, 034802 (2001).

[5] R. L. Gluckstern, Phys. Rev. Lett. 73, 1247 (1994).

[6] J. Qiang and R. D. Ryne, Phys. Rev. ST Accel. Beams 3, 064201 (2000).

[7] I. Hofmann, Phys. Rev. E 87, 4713 (1998).

[8] I. Hofmann, G. Franchetti, O. Boine-Frankenheim, J. Qiang, and R. Ryne, Phys. Rev. ST Accel. Beams 6, 024202 (2003).

[9] G. Franchetti, I. Hofmann, and D. Jeon, Phys. Rev. Lett. 88, 254802 (2002).

[10] J. Qiang, R. Ryne, S. Habib, V. Decyk, J. Comput. Phys. 163, 434 (2000).

[11] M. Reiser, "Theory and Design of Charged Particle Beams," John Wiley \& Sons, 1994, New York, NY 10158. 